\title{
Three-Dimensional Evaluation of Cervical Vertebral Morphology in Skeletal Class II of Malocclusion in Egyptians
}

\author{
Amr H. Ghazy ${ }^{*}$, Waleed E. Refaat, Sherif S. Morcos \\ Department of Orthodontics, Faculty of Dentistry, Suez Canal University, Egypt.
}

\begin{abstract}
Background: evaluation of skeletal age is of a very high importance during determining the appropriate treatment plan for orthodontic patients, growth modification isn't applicable in skeletally mature patients while it could be the treatment of choice in growing ones. That's why Cervical vertebral morphology is of great importance in the field of orthodontics as it can be used to assess skeletal maturity. Aim: to study the morphology of cervical vertebrae in individuals with skeletal Class II malocclusion. Materials and Methods: Twenty Cone-beam computed tomography images of male and female Egyptian patients aged from 18 to 40 years were studied. Patients were divided into two groups according to ANB angle: group 1, Skeletal Class I (ANB=1:3); and group 2, Skeletal Class II (ANB>3). Eight linear measurements and one angular measurement were used to evaluate the morphology of $\mathrm{C}_{1}$ vertebra. Results: The Cone-beam images of the twenty patients were evaluated to assess the morphology of the first cervical vertebra in both groups, eight linear and one angular variables were compared in both groups significant differences were found between both groups regarding the horizontal outer transverse diameter of $C_{1}$ (HOTDC1) with $P$ value of 0.026 , Lateral outer anteroposterior diameter of $C_{1}$ (LOAPC1) with $P$ value of 0.04 , Frontal outer transverse diameter of $C_{1}$ (FOTDC1) with $P$ value of 0.025 and Dens Angle with $P$ value of 0.02 . Conclusion: The morphology of the cervical vertebrae was found to be affected by the anteroposterior relation of the maxilla to the mandible.
\end{abstract}

Keywords: Cervical vertebrae, Skeletal Class I, Cone- beam computed tomography (CBCT).

\section{Introduction}

Skeletal malocclusion can be classified into Class I, Class II and Class III depending on the anteroposterior relation of the basal bones of the jaws. The treatment plan is determined depending on the growth stage of each patient; in growing patients the skeletal relation can be corrected using functional appliances, the best time for this approach is during the pubertal growth spurt and this approach is called "orthopedic treatment". While in non- growing patients the treatment can be dental camouflage of the abnormal skeletal relation and this is termed "orthodontic treatment" or in more severe cases "orthognathic treatment" must be considered as these cases can't be treated by orthodontic approach only. There are many biological indicators that can be used to determine the growth stage of each patient including Hand-Wrist radiographs and Cervical Vertebral Morphology ${ }^{(1-4)}$. It was found that the cervical vertebrae maturation stages can be used as a replacement for 
Hand-Wrist bone maturation stages for evaluation of patient maturational stage ${ }^{(5)}$. The aim of this study was to evaluate the morphology of cervical vertebrae by using Cone-beam computed tomography images ( $C B C T)$.

\section{Subjects and Methods}

Subjects: The sample of this study was consisted of CBCT scan radiographs for 20 Egyptian patients that didn't undergo previous orthodontic treatment, the radiographs were taken for those patients before starting orthodontic treatment at the Department of Orthodontics of Suez Canal University dental college. The study group included males and females without sex predilection. The subjects of this study were with age range from 18 to 40 years and were selected as 10 patients of Skeletal Class I malocclusion and 10 patients of Skeletal Class II malocclusion. The patients were considered of Skeletal Class I pattern when $(A N B=1: 3)$, and of Skeletal Class II pattern when (ANB>3).

Obtaining Cone Beam Computed Tomography CBCT Images: The cervical vertebrae were scanned and analyzed using SCANORA* 3Dx-Cone Beam CT-SORDEX of the Radiology Department at the Faculty of Dentistry of Suez Canal University (Figure 1). For obtaining the CBCT Images the patients were seated with their teeth in maximum intercuspation (centric occlusion), their heads were positioned in a way that the Frankfort and the midsagittal plane were perpendicular to the floor. They were placed in the SCANORA 3DxCone Beam CT with their facial median line vertical to the floor and Frankfort plane parallel to the floor, this position was adjusted for each patient and controlled by a guideline directed from the front and the sides. CBCT images were used to evaluate the maxillofacial characteristics of Class I and Class II subjects. SNA; SNB and ANB angles were measured using $C B C T$ images.

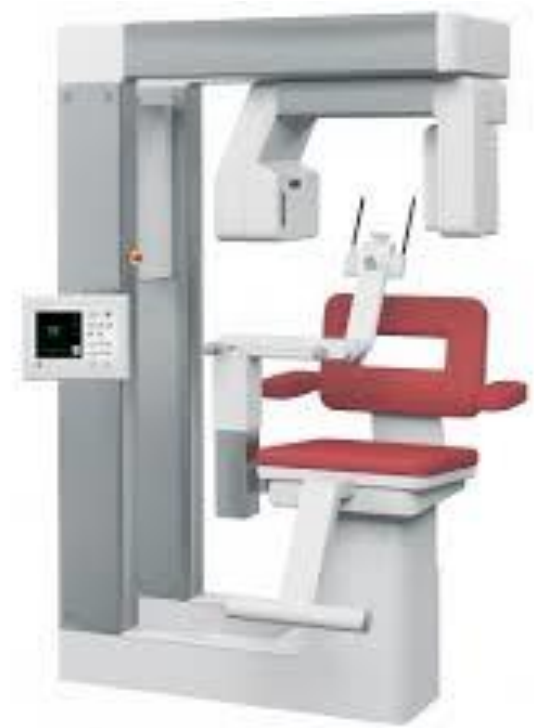

Figure 1: Photograph showing SCANORA 3Dx-Cone Beam CT- SORDEX

Measurements(6): In this study we used eight linear measurements and one angular measurement to assess the morphology of cervical vertebrae with different sagittal skeletal pattern. 1) Horizontal inner and outer anteroposterior (AP) diameter of the first cervical vertebra (C1) $(\mathrm{mm})$ (HOAPC1; Figure 2). 2) Horizontal outer transverse diameter of $\mathrm{C}_{1}(\mathrm{~mm})$ (HOTDC1; Figure 2). 3) Distance between outer margin of transverse foramen and outer margin of lateral mass $(\mathrm{mm})$ (outer margin; Figure 2). 4) AP diameter of superior surface of $\mathrm{C} 1$ anterior arch $(\mathrm{mm})$ (superior surface; Figure 2). The Lateral outer AP diameter of $\mathrm{C} 1(\mathrm{~mm})$ (LOAPC1) as well as the height of the atlas dorsal arch ( $\mathrm{mm}$ ) (dorsal arch) is shown in figure (3). The frontal outer transverse diameter of $\mathrm{C}_{1}(\mathrm{~mm})$ (FOTDC1) is shown in figure (4). The angle along axis line of the dens to occlusal plane (dens angle [degrees]) is shown in figure (5).

\section{Statistical analysis}

Data were tabulated, coded then analyzed using the computer program SPSS (Statistical package for social science) version 17.0 for Windows. Descriptive statistics, including means, standard deviations and t-test with statistical significance level 95\%, level of confidence $(p<0.05)$. 


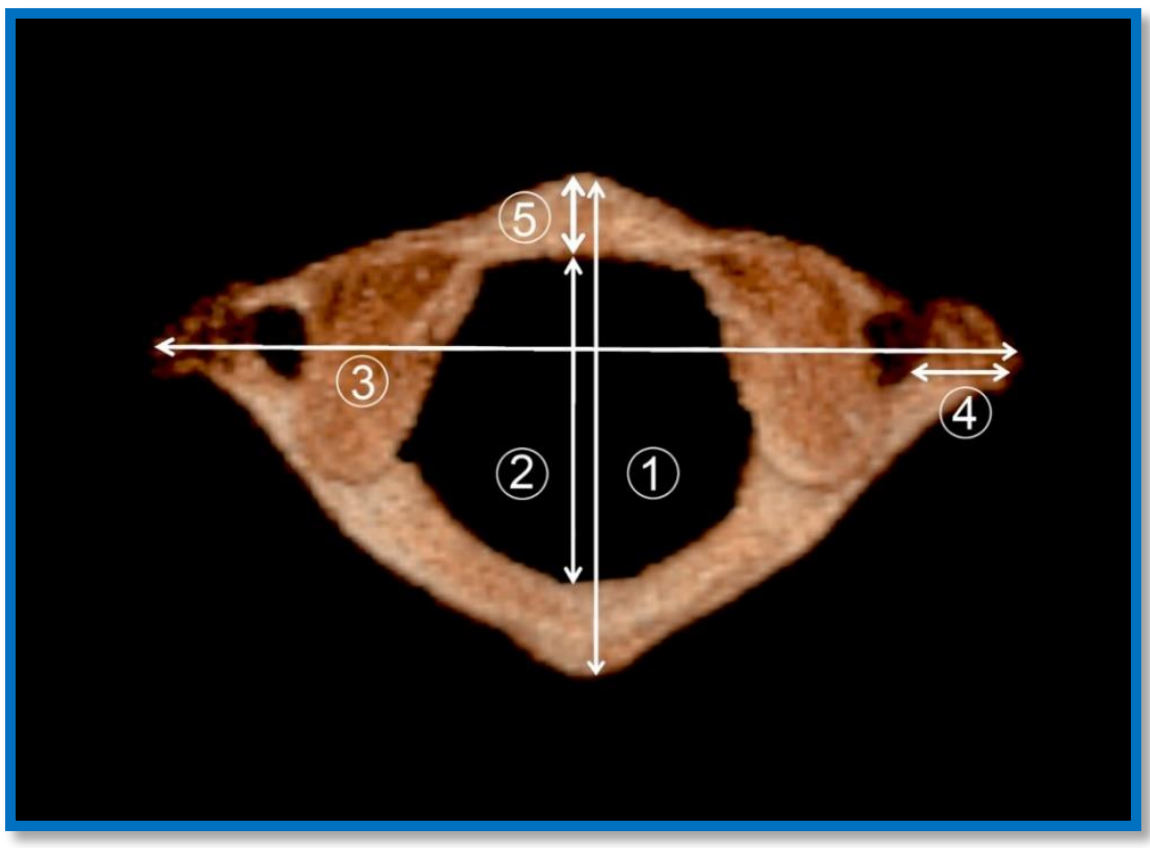

Figure 2: axial view of second cervical vertebra

(1) Horizontal outer anteroposterior (AP) diameter of the first cervical vertebra (C1) (mm) (HOAPC1). (2) Horizontal inner anteroposterior diameter of $\mathrm{C}_{1}(\mathrm{~mm})$ (HIAPC1). (3) Horizontal outer transverse diameter of $\mathrm{C}_{1}(\mathrm{~mm})$ (HOTDC1). (4) Distance between outer margin of transverse foramen and outer margin of lateral mass ( $\mathrm{mm})$ (outer margin). (5) AP diameter of superior surface of C1 anterior arch ( $\mathrm{mm}$ ) (superior surface).

\section{Results}

The mean value of cervical vertebra measurements in Class I and Class II groups is shown in table (1). It was found that there are significant differences in "horizontal outer transverse diameter of $\mathrm{C}_{1}$ ", "lateral outer anteroposterior diameter of $\mathrm{C}_{1}$ ", "frontal outer transverse diameter of $\mathrm{C}_{1}$ "and "Dense angle" between Class II and Class I patients.

Table 1: The mean value of cervical vertebra measurements

\begin{tabular}{|l|cc|cc|c|}
\hline \multirow{2}{*}{} & \multicolumn{2}{|c|}{ CLASS I } & \multicolumn{2}{c|}{ CLASS II } & \\
\cline { 2 - 6 } & Mean & \pm SD & Mean & \pm SD & $P$ \\
\hline HOAPC1 & 44.14 & 3.82 & 41.57 & 2.91 & 0.1 \\
\hline HIAPC1 & 27.87 & 3.18 & 26.63 & 2.27 & 0.33 \\
\hline HOTDC1 & 74.44 & 4.40 & 70.16 & 3.42 & $0.026^{*}$ \\
\hline Outer margin & 8.43 & 1.65 & 7.85 & 1.27 & 0.38 \\
\hline Superior surface & 7.29 & 1.42 & 6.53 & 1.30 & 0.23 \\
\hline LOAPC1 & 45.01 & 3.24 & 41.66 & 3.57 & $0.04^{*}$ \\
\hline dorsal arch & 8.64 & 1.60 & 9.21 & 1.38 & 0.4 \\
\hline FOTDC1 & 74.21 & 4.47 & 69.79 & 3.54 & $0.025^{*}$ \\
\hline dense angle & 96.55 & 7.31 & 88.04 & 7.86 & $0.02^{*}$ \\
\hline
\end{tabular}

HOAPC1: horizontal outer anteroposterior diameter of C1; HIAPC1: horizontal inner anteroposterior diameter of C1; HOTDC1: horizontal outer transverse diameter of C1; LOAPC1: lateral outer anteroposterior diameter of C1; FOTDC1: frontal outer transverse diameter of $\mathrm{C}_{1}$; SD: standard deviation, P: Probability; *: significance $\leq 0.05$; Test used: Student's t-test(Unpaired) 


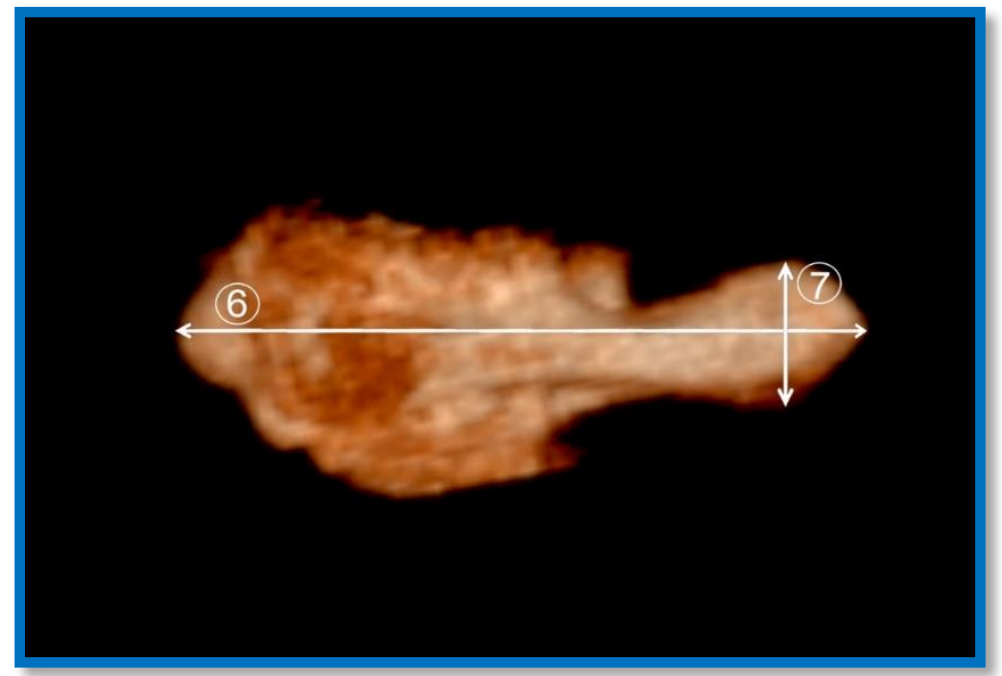

Figure 3: Lateral view of second cervical vertebra.

No. (6) = the lateral outer AP diameter of $C_{1}(\mathrm{~mm})$ (LOAPC1).

No. $(7)=$ Height of the atlas dorsal arch $(\mathrm{mm})$ (dorsal arch).

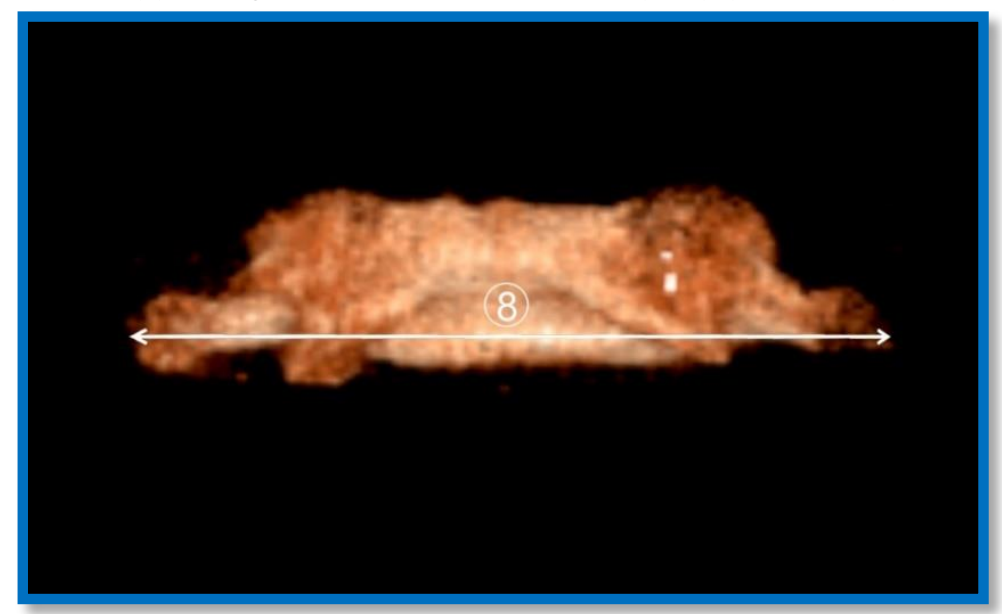

Figure 4: Frontal view of second cervical vertebra No. $(8)=$ the frontal outer transverse diameter of $\mathrm{C}_{1}(\mathrm{~mm})($ FOTDC1)

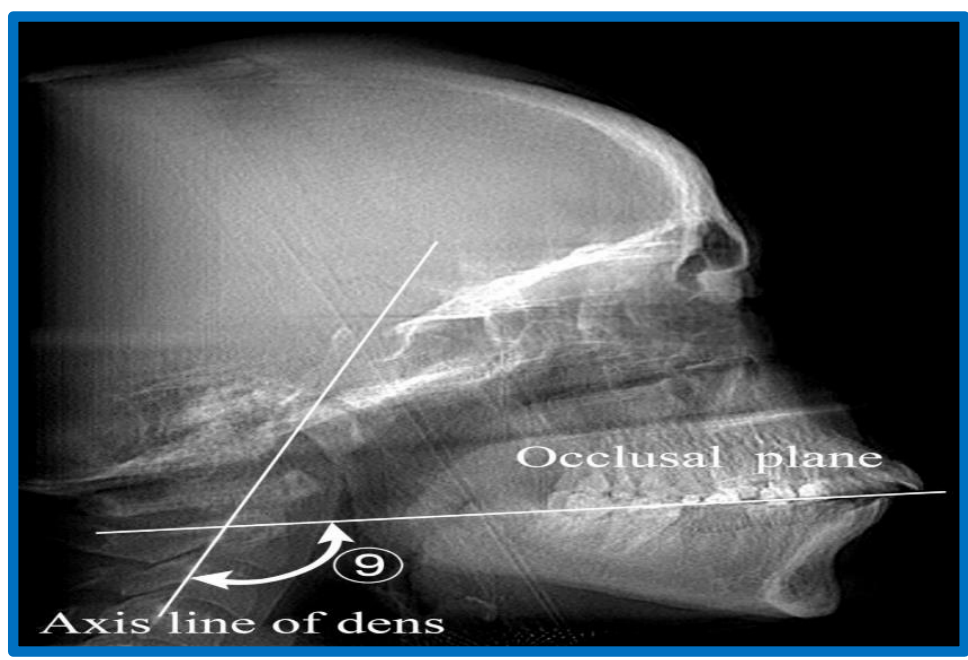

Figure 5: Lateral cephalometric image extracted from CBCT No. (9) = the angle along axis line of the dens to occlusal plane (dens angle [degrees]). 


\section{Discussion}

The cervical vertebral morphology is used to assess skeletal maturity in orthodontic patients and according to the different skeletal patterns the treatment plan is selected, in growing patients the treatment of choice is growth modification while in non-growing ones the treatment of choice is either orthodontic or orthognathic treatment depending on the severity of the case. The present study was designed to evaluate the cervical vertebral morphology in skeletal Class II maloccclusion Egyptians compared with the morphology of the vertebrae in Class I patients using "CBCT" imaging in this study nine variables were used to assess the morphology of the first cervical vertebrae, it was found that the horizontal outer transverse diameter, the lateral outer anteroposterior diameter, the frontal outer transverse diameter and Dense angle showed significant differences between both groups. It can be concluded that significant differences were found between the morphology of $\mathrm{C}_{1}$ in Skeletal Class II patients when compared with the morphology of $\mathrm{C}_{1}$ in patients with Skeletal Class I malocclusion, this was found to be in accordance with many studies that were performed to evaluate the morphology of cervical vertebrae in patients with different Skeletal Classes and in patients with certain medical conditions ${ }^{(6-}$ 17).

\section{Conclusion}

The following conclusions could be drawn from the current study: 1 . Our study confirmed past findings that cervical vertebral morphology varies among individuals with different anteroposterior skeletal patterns. 2. This study found that there was no significant difference in terms of dorsal arch height among Egyptian individuals with different anteroposterior skeletal patterns. 3. There were significant differences between "HOTDC1","LOAPC1", "FOTDC1" and "Dens angle" among individuals with different anteroposterior skeletal patterns. 4. The measures were found to be significantly decreased in Class II patients relative to Class I.

\section{References}

1. Hassel B, Farman AG. Skeletal maturation evaluation using cervical vertebrae. Am J Orthod Dentofacial Orthop 1995;107(1):58-66.

2. Franchi L, Baccetti T, McNamara JA, Jr. Mandibular growth as related to cervical vertebral maturation and body height. Am J Orthod Dentofacial Orthop 2000; 118 (3):335-40.

3. Baccetti T, Franchi L, McNamara JA Jr. An improved version of the cervical vertebral maturation (CVM) method for the assessment of mandibular growth. Angle Orthod 2002; 72(4): 31623.

4. Baccetti T, Franchi L, McNamara Jr. JAM. The Cervical Vertebral Maturation (CVM) Method for the Assessment of Optimal Treatment Timing in Dentofacial Orthopedics. Seminars in Orthodontics 2005;11 (3):119-29.

5. Lai EH, Liu JP, Chang JZ, et al. Radiographic assessment of skeletal maturation stages for orthodontic patients: hand-wrist bones or cervical vertebrae? J Formos Med Assoc 2008; 107(4):316-25.

6. Watanabe M, Yamaguchi T, Maki K. Cervical vertebra morphology in different skeletal classes. A three-dimensional computed tomography evaluation. Angle Orthod 2010; 80 (4): 531-6.

7. Sonnesen L, Kjaer I. Cervical column morphology in patients with skeletal Class III malocclusion and mandibular overjet. Am J Orthod Dentofacial Orthop 2007;132 (4):427.e7-12.

8. Sonnesen L, Kjaer I. Anomalies of the 
cervical vertebrae in patients with skeletal Class II malocclusion and horizontal maxillary overjet. Am J Orthod Dentofacial Orthop 2008; 133 (2): 188.e15-20.

9. Bedoya A, Landa Nieto Z, Zuluaga LL, Rocabado M. Morphometry of the cranial base and the cranial-cervicalmandibular system in young patients with type II, division 1 malocclusion, using tomographic cone beam. Cranio 2014;32(3):199-207.

10. Baydas B, Yavuz I, Durna N, Ceylan I. An investigation of cervicovertebral morphology in different sagittal skeletal growth patterns. Eur J Orthod 2004; 26 (1):43-9.

11. Arntsen T, Sonnesen L. Cervical vertebral column morphology related to craniofacial morphology and head posture in preorthodontic children with Class II malocclusion and horizontal maxillary overjet. Am J Orthod Dentofacial Orthop 2011; 140 (1):e1-7.

12. Meibodi SE, Parhiz H, Motamedi MH, Fetrati A, Meibodi EM, Meshkat A. Cervical vertebrae anomalies in patients with class III skeletal malocclusion. J Craniovertebr Junction Spine 2011; 2(2):73-6.

13. Arslan SG, Dildeș N, Kama JD. Cephalometric Investigation of First Cervical Vertebrae Morphology and Hyoid Position in Young Adults with Different Sagittal Skeletal Patterns. Scientific World J 2014; 2014: 159784.

14. Patcas R, Tausch D, Pandis N, et al. Illusions of fusions: assessing cervical vertebral fusion on lateral cephalograms, multidetector computed tomographs, and cone-beam computed tomographs. Am J Orthod Dento-facial Orthop 2013; 143 (2): 213-20.

15. Sonnesen L, Jensen KE, Petersson AR, Petri N, Berg S, Svanholt P. Cervical vertebral column morphology in patients with obstructive sleep apnoea assessed using lateral cephalograms and cone beam CT. A comparative study. Dentomaxillofac Radiol 2013; 42 (6): 20130060.
16. Sonnesen L, Petri N, Kjaer I, Svanholt P. Cervical column morphology in adult patients with obstructive sleep apnoea. Eur J Orthod 2008;30(5):521-6.

17. Hosseinzadeh Nik T, Janbaz Aciyabar P. The Relationship Between Cervical Column Curvature and Sagittal Position of the Jaws: Using a New Method for Evaluating Curvature. Iran J Radiol 2011;8(3):161-6. 